
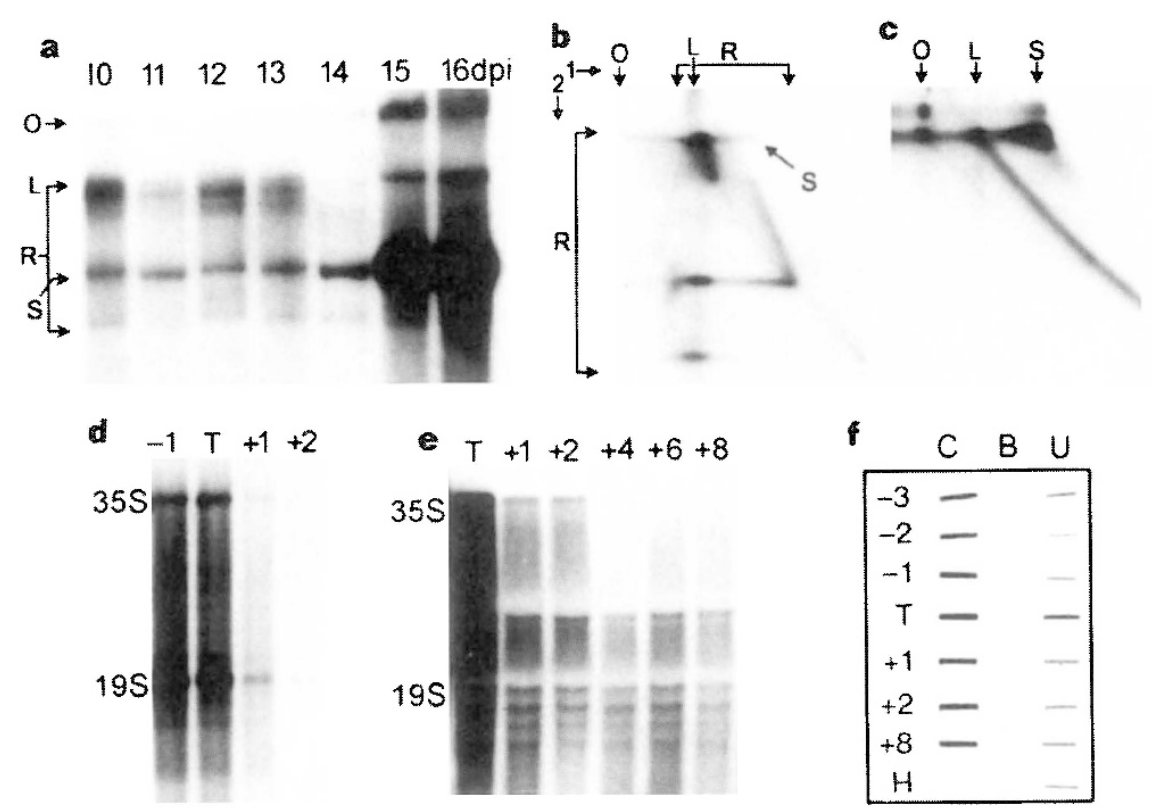

Figure 1 Changes in CaMV replication products. a, Time-course (days post-inoculation, dpi) of unencapsidated viral DNAs preceding host recovery, showing heterogeneous replication intermediates $(R)$, supercoiled (S), linear (L), and open circular (O) DNA forms. b, 2D gel separation (first dimension (I), neutral; second dimension (2), denaturing electrophoresis) of viral DNA forms showing heterogeneous growing DNA strands (R) and a low level of supercoiled DNA (S). c, 2D gel separation of post-replicative viral DNA forms. $d$, Degradation of the two main CaMV polyadenylated RNAs (35S and 19S RNA) over 2 days after the transition point $(T)$ in viral DNA forms. $e$, Total viral RNA indicating persistence of nonpolyadenylated RNA fragments. f, Nuclear run-on transcription assay from 3 days before until 8 days after the transition point. Radioactive RNA synthesized in isolated nuclei from infected or healthy $(\mathrm{H})$ plants was used to probe filters containing CaMV (C), bacterial (B) and ubiquitin (U) DNA.

total cell RNA fraction, but non-polyadenylated fragments persisted (Fig. le) indicating that viral polyadenylated RNAs were being targeted specifically for degradation by de-adenylation. However, neither viral RNA synthesis from the CaMV minichromosome nor that of a constitutivelyexpressed host gene (ubiquitin), measured by run-on transcription in isolated nuclei, showed any significant change up to 8 days after the transition (Fig. 1f). These changes in the levels of viral intermediates just before plant recovery are consistent with a sudden arrest of viral replication by gene silencing, because continued transcription seen with RNA degradation is a hallmark of post-transcriptional silencing found in a variety of guises in transgenic plants ${ }^{1-5}$.

We detected differences in the timing of the replicative transition in different parts of the same plant, implying that the response is not elicited at the whole-plant level. As there is amplification of the CaMV minichromosome it is likely that the viral replication cycle has become deregulated, allowing retargeting of progeny viral genomes back to the nucleus. Most probably, retargeting is prevented by a viral factor synthesized during replication. The unchanged rate of transcription suggests that most of the newly amplified minichromosome DNA is inactive. We have found differences in the population of superhelical topoisomers present but no differences in DNA methylation.

It is not clear whether DNA amplification is the cause or consequence of gene silencing. If silencing is initiated by host recognition of viral RNA as a target for degradation, then amplification of the minichromosome would result from loss of the factor preventing nuclear retargeting. Alternatively, amplification could itself initiate silencing through an ectopic interaction' between transcriptionally inactive and active minichromosomes. Consistent with both models, we have observed silencing of a reporter transgene with homology to CaMV RNAs but only in tissues showing DNA amplification where viral replication had ceased (unpublished results).

If, as we suggest, gene silencing underlies this natural resistance to virus infection, is antiviral defence one part of a multifaceted mechanism regulating plant gene expression, or is transgene silencing a consequence of the way that plants combat viruses? Whichever is the case, unravelling the early molecular steps should provide new opportunities for manipulating genes and controlling plant viral diseases.

Simon N. Covey, Nadia S. Al-Kaff, Amagoia Lángara, David S. Turner Department of Virus Research,

John Innes Centre, Norwich Research Park,

Colney, Norwich NR4 7UH, UK

e-mail:covey@bbsrcac.uk
1. Baulcombc, D. C. \& English, J. E. Curr. Opin. Biotechnol. 7, $173-180(1996)$.

2. Baulcombe, D. C. Plant Cell 8, 1833-1844 (1996).

3. Flavell, R. B. Proc. Natl Acad. Sci. USA 91, 3490 - 3496 (1994).

4. Matzke, M. A. \& Matzke, A. I. M. Plant Physiol. 107,6679-6685 (1995).

5. Meyer, P. \& Sadler, H. Annu. Rev. Plant Physiol. Plant Mol. Biol. 47, 23-48 (1996).

6. Rothnie, H. M., Chapdelaine, Y. \& Ilohn, T. Adv. Virus Res. 44, $1-67$ (1994).

7. Al-Kaff, N. S. \& Covcy, S. N. Plant Pathol. 44, 516-526 (1995).

8. Covey, S. N., Turner, D. S., I.ucy, A. I. \& Saunders, K. Proc. Nat Acad. Sci. USA 87, 1633-1637(1990).

\section{Evening carbon atom oddities}

In their Scientific Correspondence, J. Sarma et al. (Nature 384, 320; 1996) seem to show more than a simple 'even-odd carbon atom disparity'. Many of the naturally occurring organic compounds containing between 40 and 80 carbon atoms (and indeed in the frequency distribution peak of the Cambridge Structural Database; $d$ in their figure) are undoubtedly derivatives either of hexose sugars or of aromatic rings.

The periodicity of compounds with a multiple of six carbon atoms is striking ( $\mathrm{c}$ in their figure). In addition, many synthetic organic compounds are derived from natural (including petrochemical) sources, and therefore would reflect this natural periodicity bias in the range 6-80 carbon atoms (shown in parts $a$ and $b$ ).

Acetate (with 2 carbon atoms), perhaps one of the prebiotic molecules synthesized from methane and formaldehyde (see S. Miller, Science 117,$528 ; 1953$ ) is also one of the primary building blocks of cellular organic compounds.

\section{Matthew Kaser}

Department of Pediatrics,

University of California at San Francisco,

1001 Potrero Avenue,

San Francisco,

California 94110, USA

e-mail:mkaser@sfghpeds,ucsf.edu

Sarma et al. (Nature 384, 320; 1996) make the interesting observation that there are more organic compounds with an even number of carbon atoms than with an odd number. The pronounced excess of $\mathrm{C}$-even compounds among those with large numbers of carbon atoms could be explained if a significant proportion of the compounds are dimers or other polymers, as any polymer of a C-even compound can, of course, only be $\mathrm{C}$-even, whereas polymers of $\mathrm{C}$-odd compounds can be C-even or C-odd.

Peter G. Stanley

Department of Biochemistry

and Molecular Biology,

University College London,

Gower Street,

London WCIE 6BT, UK 